\title{
A semiautomatic method for adding behavioral data to videotape records in combination with the Noldus Observer system
}

\author{
ANDREAS BISCHOF, HOLGER STARK, REINHARD BLUMENSTEIN, THOMAS WAGNER, \\ ANDRÉ BRECHMANN, and HENNING SCHEICH \\ Leibniz Institute for Neurobiology, Magdeburg, Germany
}

\begin{abstract}
The Observer 3.0 (Noldus, 1991) was used as the base for a time-saving fine analysis of animal behavior. A PC controlled a shuttlebox and a shuttlebox control unit. Learning behavior was recorded on videotapes by an S-VHS camcorder with vertical interval time code (VITC), which was used to generate an observational data file (ODF). The observational system was enhanced by automatically recorded status signals from the PC as event markers on the soundtrack of the videotape. These signals were used to subdivide a training session into numbered trials during generation of the ODF. They were later used for a PC-aided fine analysis of behavioral events, such as attention responses (AR) and orienting responses (OR) during shuttlebox learning. In this way, a large number of both automatically inserted PC event markers and manually inserted, key-defined behavioral events were registered and analyzed effectively in a semiautomatic fashion. The system is illustrated with an analysis of shuttlebox avoidance data from gerbils (Meriones unguiculatus).
\end{abstract}

Often, researchers must coordinate several behavioral measures or additional data streams with videotape records. The present system provides a straightforward way to write data directly onto a videotape from the PC that controlled the learning experiment in real time and to extract these data synchronized with the videotape record. This system takes advantage of the videotape recorder's time coded track and its two audio channels (not available on low-end VHS) and allows researchers to record PC status signals, such as the onsets of conditioned stimulus (trial onsets) and unconditionedstimulus, as well as animals' response events during shuttlebox learning. Otherwise, these important schedule components would not be apparent to the observational data file (ODF), and the key-defined insertion of trial onsets and animal responses using the keyboard of the analysis PC would have to be performed manually (and time-consumingly) by a person watching the videotape during behavioral analysis.

Studying animal learning merely on the base of particular behavioral measurements (i.e., the rate of conditioned responses) might lead to an incomplete conclusion. Thus, the observation of several further behavioral events can aid in a more complete understanding of learning mechanisms.

\section{METHOD}

The Experimental Setup

This setup consisted of a shuttlebox, a PC, a shuttlebox control unit with a DTMF coder, and a video camera (Figure 1A).

The authors thank Karin Schmidt for skillful technical assistance. Correspondence should be addressed to H. Stark, Department of Auditory Plasticity and Speech, Leibniz Institute for Neurobiology, P. O. Box 1860, 39008 Magdeburg, Germany (e-mail: stark@ifn-magdeburg.de).
The shuttlebox was controlled by the PC via a shuttlebox control unit that was connected to the PC by a digital I/O card. The presentation of the tone stimuli and the delivery of electric footshock pulses to the grid floor of the shuttlebox were PC controlled. In parallel, the PC status signals, such as tone stimulus onset, footshock onset, and animal response, were transformed into the exceptionally noise-resistant dual-tone multifrequency (DTMF) code (Figure 2) and recorded on a videotape by a camcorder (Figure 3). DTMF is the touch-tone sound that is familiar to telephone users.

During the shuttlebox experiment, the data logging apparatus Panasonic AG-DP200E S-VHS camcorder recorded the behavior of each animal and a vertical interval time code (VITC). This is a standard code that numbers each frame of the videotape. It is available on $\mathrm{S}$ VHS, 8-mm, Betacam, and some U-matic recorders. Eight status signals from the PC that controlled the shuttlebox were transformed by the DTMF code (Figure 2) and inserted on the left soundtrack of the videotape during the training. These tone-coded markers included the trial start (onset of the footshock-announcing tone) and the animal's response (changing the shuttlebox compartment). In this way, these important triggers of the animal's reaction time were automatically coded. Up to 16 PC status signals as event markers could be recorded onto videotape.

The other available right soundtrack could be used for recording the sounds in the shuttlebox, (i.e., the tone stimuli and animal vocalization) or for encoding further important events of the behavioral experiment.

A monolithic DTMF transceiver MT8889C (Mitel) was adapted for generating the DTMF code. The input impulses to the DTMF coder (located in the shuttlebox control unit) were delivered by an 8255 based digital I/O card of the control PC. The output of the transceiver was connected with the line input of the camcorder (Figures $1 \mathrm{~A}$ and 2). The transceiver circuit was initialized and controlled by the shuttlebox software.

\section{The Analysis Setup}

This setup consisted of S-VHS video recorder, a monitor, a DTMF decoder, and a PC for analyzing the behavioral experiments (Figure 1B). 
(A) EXPERIMENTAL APPARATUS

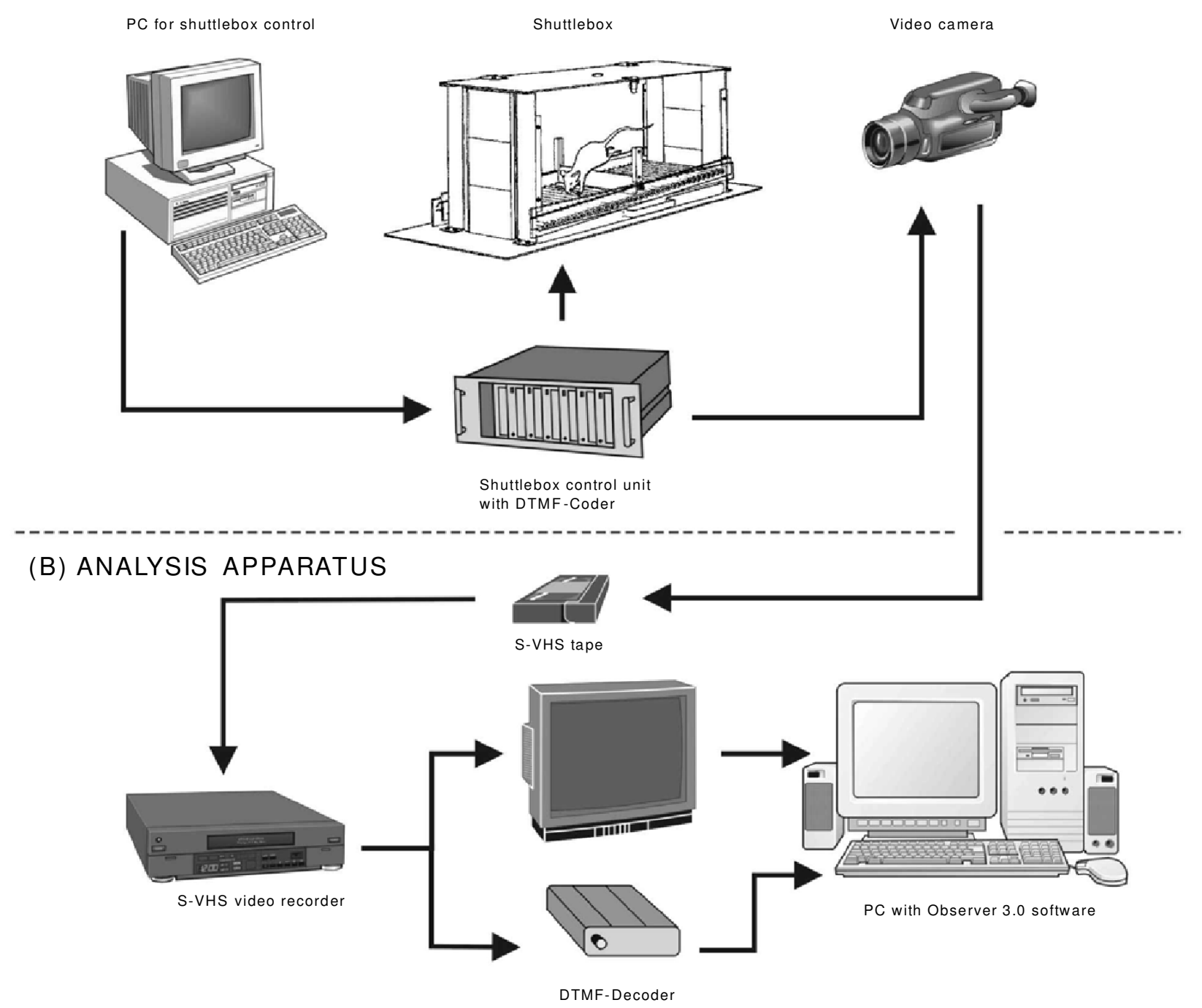

Figure 1. Experimental and analysis apparatus.

After the behavioral experiments, the videotape, on which the animal behavior and the DTMF tone-coded PC status signals were recorded, was monitored by the person who analyzed the behavior during shuttlebox learning, using a video recorder. Observer's codes for the specific time-coded behavioral events, such as AR and OR, were manually entered into the analysis PC during the running of the Noldus database program. Each behavioral event was marked as a key code by pressing the appropriate key on the keyboard of the analysis PC. The Noldus database software collected this information and transferred it into the ODF (Figures 1B and 5) (Noldus, 1991; Noldus, Trienes, Hendriksen, Jansen, \& Jansen, 2000). During this procedure, up to 84 behavioral events could be manually entered using the keyboard, and up to eight DTMF-coded signals could be automatically inserted.

For decoding of the DTMF signals, we used a DTMF transceiver MT8889C. The decoder was built as a small device, containing the MT8889C and a BASIC-Stamp II Module (Parallax, Inc., 599 Menlo Dr., Suite 100, Rocklin, CA 95765), whose input was connected to the video recorder (Figure 4). The BASIC-Stamp II Module is a simple one-chip microcontroller programmable in a BASIC subset, with 16 digital in/outputs.

The DTMF transceiver was connected to seven output pins of the BASIC-Stamp. The interface to the analysis PC keyboard was composed of eight relays triggered by the outputs of the microcontroller. The contacts of these relays were connected in parallel to eight predefined keys of the keyboard. A BASIC program running in the Stamp module initialized the DTMF transceiver, and, in this way, the relays were related to the decoded tones. As a result, eight decoded tones simulated eight defined keystrokes. The limitation to 8 of 16 tones is nonrelevant for this presentation. An upgrade to the full range of 16 tones is possible by modification of the BASIC program and some additional hardware logic.

\section{Evaluation of the Behavioral Experiment}

During monitoring, the ODF generated by the Noldus program was automatically imported by Visual Basic macros (Microsoft Excel 5.0). The key-defined PC status signals and the manually defined behavioral events, all VITC coded, such as tone onsets, footshock on- 


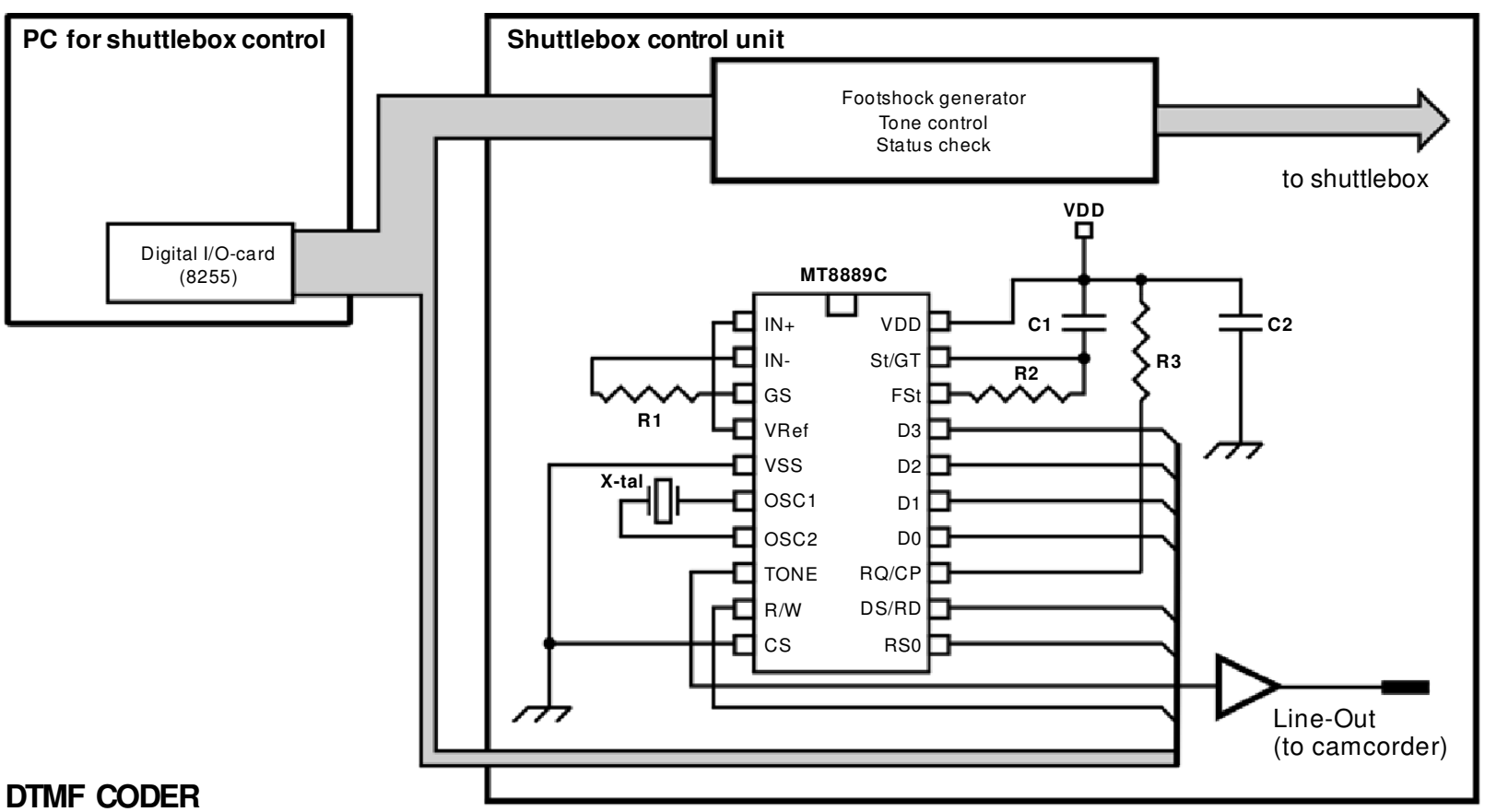

Figure 2. Schematic block diagram of the dual-tone multifrequency (DTMF) coder using the Mitel monolithic DTMF transceiver MT8889C.

sets, animal responses, attention responses, and orienting responses, were analyzed trial by trial of the training session as well as from trial onsets up to the end of trials (Figures 5,7). For this, the Excel macros used the automatically inserted event markers for tone onsets (trial onset) and gerbil's compartment changes to calculate, for example, the conditioned responses (in case of compartment change before footshock onset). After this analysis, the Excel macros automatically generated diagrams, which allowed us to follow the development of

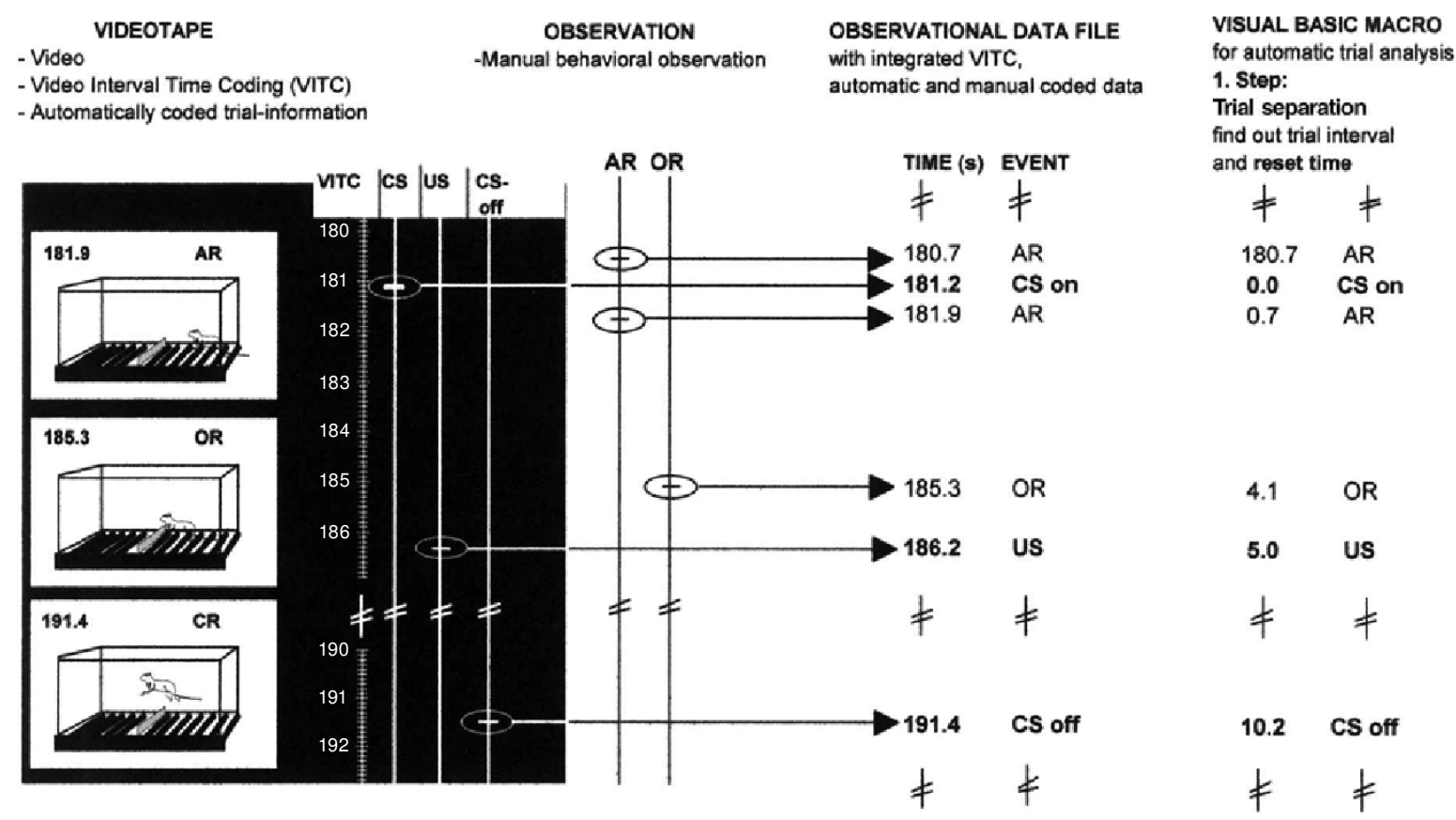

Figure 3. Integration of automatically recorded PC event markers and manually inserted observations from a shuttlebox experiment and transformation into an observational data file (ODF). 


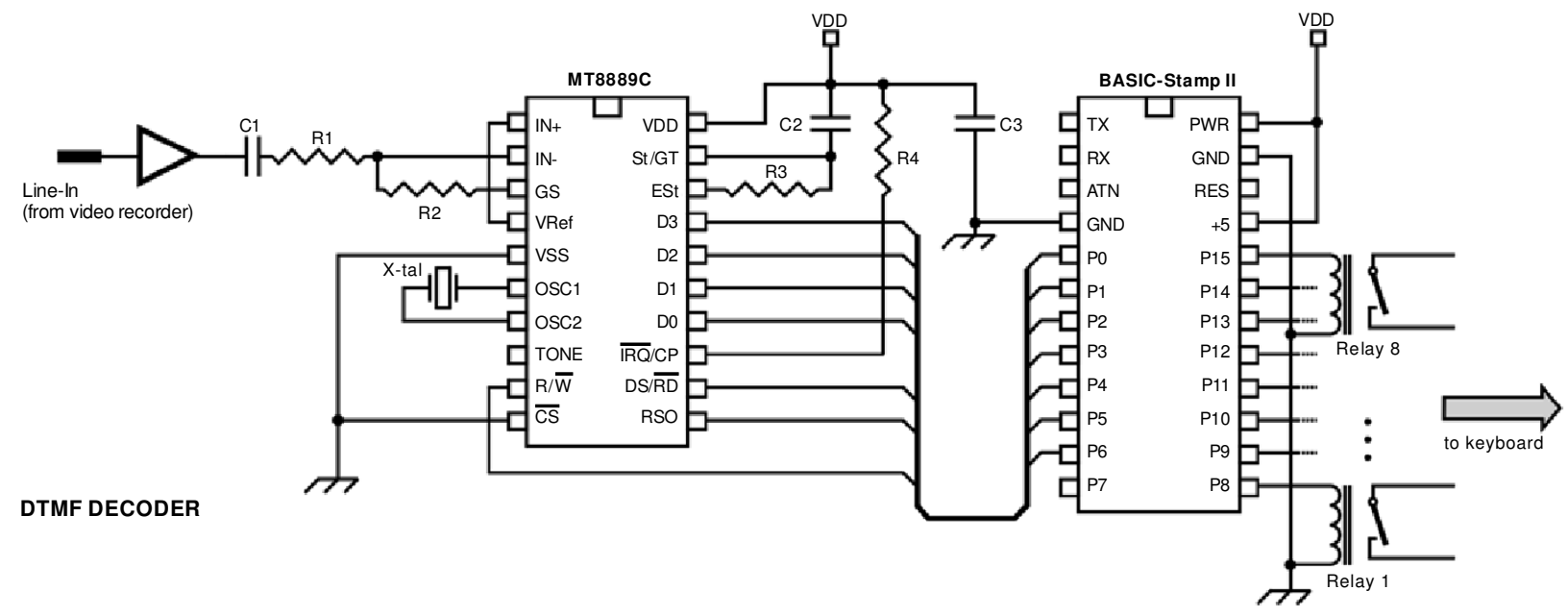

Figure 4. Schematic block diagram of the dual-tone multifrequency decoder consisting of a Mitel DTMF transceiver MT8889C and a BASIC-Stamp II from Parallax, Inc.

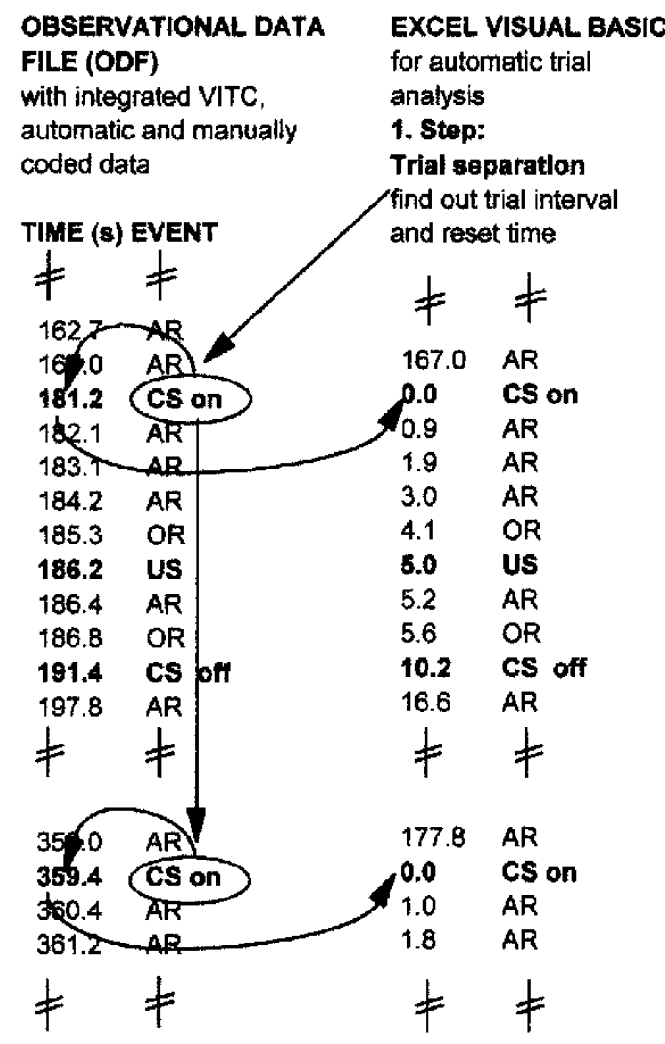

\author{
EXCEL VISUAL BASIC \\ for automatic trial analysis \\ Following Steps: \\ (realized by logic functions in \\ Excel Visual Basic Macro)
}

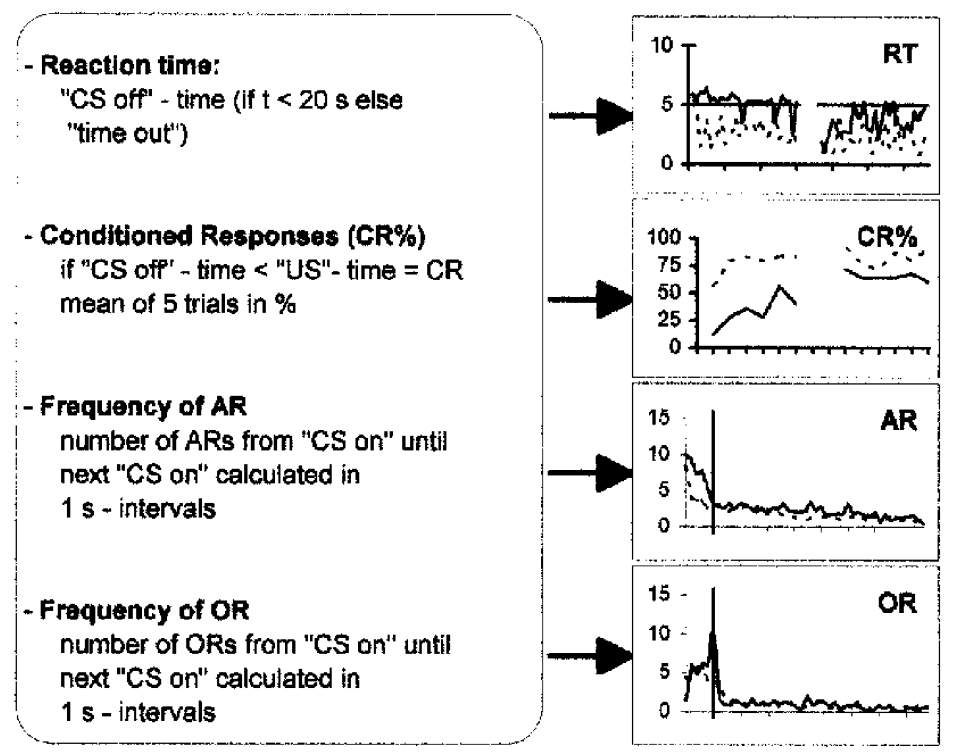

Figure 5. Analysis of PC-generated and Observer-generated data streams by custom-made macros programmed in Excel Visual Basic. The Excel macros sorted PC status signals from the shuttlebox and the continuously stored behavioral events into numbered trials based on the VITC-coded impulses for trial onset. The times of occurrence of the PC impulses and the key-coded behavioral events were rescaled to the corresponding trial onset times. These files simplified the analysis of the data. AR, attention response; $O R$, orienting response; CR, conditioned response; CS, conditioned stimulus; US, unconditioned stimulus. 
1. AVOIDANCE TRAINING

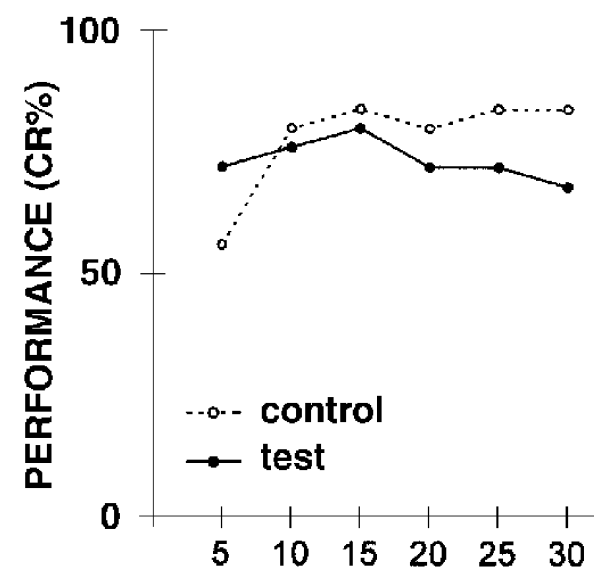

\section{AVOIDANCE} TRAINING

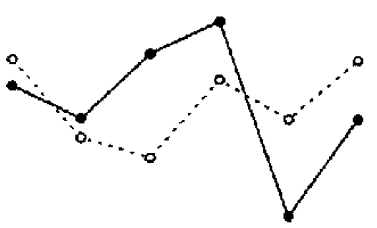

\section{TRIALS}

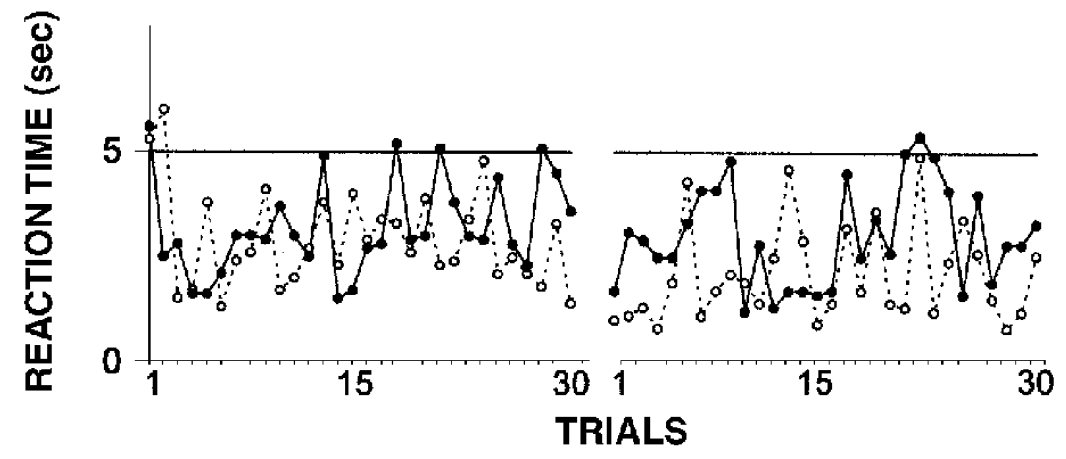

Figure 6. The upper frame depicts the averaged learning curves during two consecutive avoidance training sessions for the avoidance control group $(\bigcirc, n=5)$ and a test group subjected to a preexperience $(\Theta, n=5)$. No significant differences were found in the avoidance performance. $C R$, conditioned response. The lower frame depicts mean reaction times from the trial onset to change of shuttlebox compartment of the control group and the test animals. No differences were found.

conditioned responses (Figure 6) and the development of behavioral events, such as the AR during the course of the sorted trials within a training session (Figure 7).

\section{Shuttlebox Procedure}

Briefly, the shuttlebox (Coulbourn Instruments) consisted of two compartments that were separated by a barrier. This box was placed within a sound-attenuating chamber.

The experimental procedure as an avoidance paradigm presented to the gerbils a frequency-modulated sine tone $(0.9-10 \mathrm{kHz}$, modulation frequency $2.0 \mathrm{~Hz}, 60 \mathrm{~dB}$ SPL) as an auditory conditioned stimulus (CS). The tone was followed, after $5 \mathrm{sec}$, by the unconditioned footshock (unconditioned stimulus, US), which was generated from a constant-current source of the control unit $(0.6-\mathrm{mA}$ pulses for a maximum of $15 \mathrm{sec}$ via a grid floor). Every $0.33 \mathrm{sec}$, the polarity of the grid rods in the shuttlebox was changed. Tone and shock stopped as soon as the gerbil changed shuttlebox compartments. The reaction time, calculated by Excel macros as time interval from onset of conditioned stimulus until change of shuttlebox compartment, was automatically tone-coded by PC signals. In this way, during an avoidance learning paradigm, a compartment change within $5 \mathrm{sec}$ after tone onset (i.e., before the onset of the footshock) was also rated automatically as an avoidance response during the ODF analysis using Excel macros.

For the behavioral fine analysis of shuttlebox learning, further behavioral events were considered (such as the AR as a short arrest of ongoing behavior and the OR as an orientation of the animal toward the other shuttlebox compartment) as indicators of motor response preparation, but not the execution itself. AR and OR were manually key-defined and inserted into the analysis PC by the person analyzing the videotapes. In this way, both behavioral events were timecoded and transferred into the ODF (Figure 2B).

\section{RESULTS}

For validation of the experimental setup and the analysis setup described above, we demonstrate an evaluation of a shuttlebox experiment. The following behavioral parameters from the ODF were analyzed: (1) the avoidance performance (i.e., the percentage of conditioned responses to 


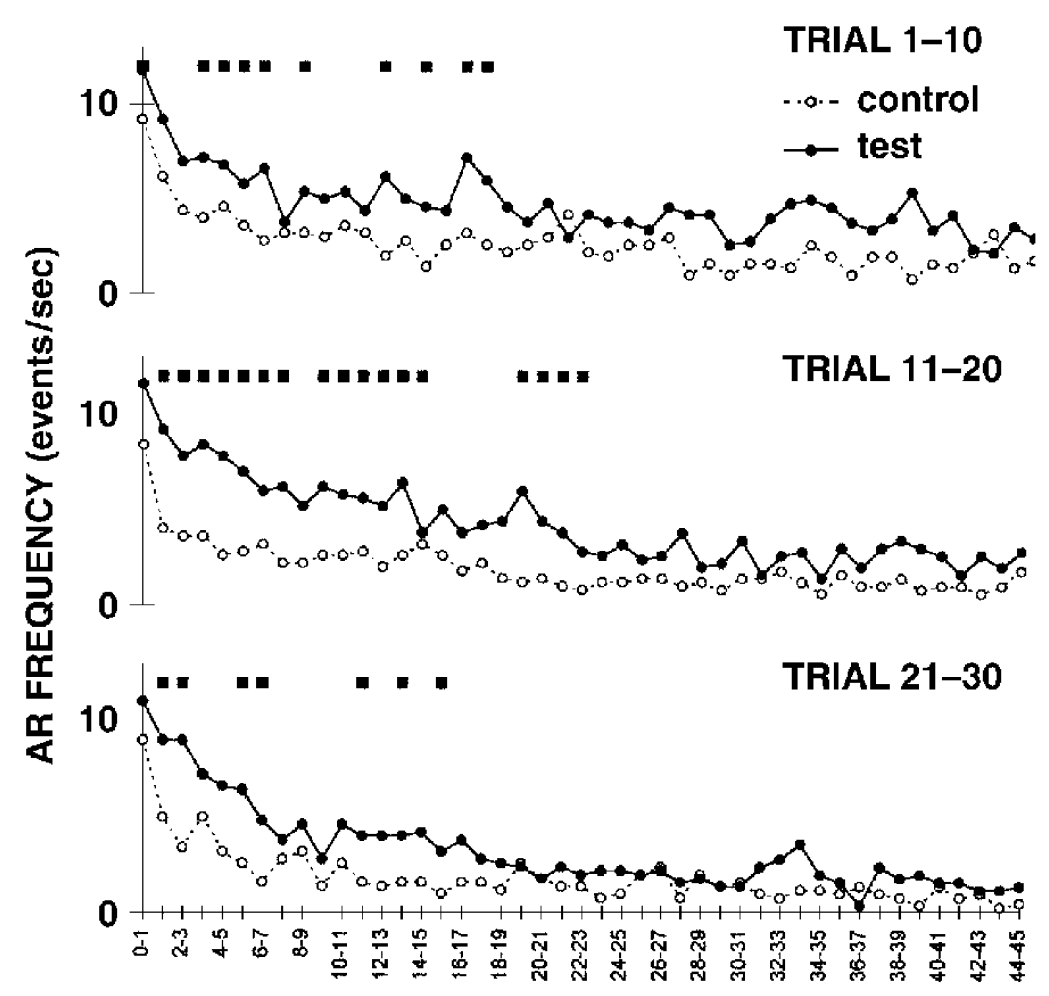

TIME (sec)

Figure 7. Averaged frequencies of attention responses (AR) between 0 and $45 \mathrm{sec}$ after trial onset separately for Trial Intervals 1-10, 11-20, and 21-30 of the control group $(O)$ and the test group (๑) during the first avoidance training session. The filled rectangles indicate the periods of significant differences between these groups (least significant difference test, $p \leq \mathbf{. 0 5}$ ). Data are mean values.

the conditioned stimuli CS; mean of 5 trials), (2) the reaction time, (3) the AR after tone onset in terms of movement arrest as a sign of signal detection and information processing, and (4) the OR toward the hurdle as a first indicator of initiation of the motor response (not demonstrated here).

The control group $(n=5)$ was composed of naive animals that were subjected to an avoidance paradigm with two training sessions on 2 consecutive days. The CS tone was followed, after $5 \mathrm{sec}$, by the US, an unconditionedfootshock. Tone and shock were interrupted by a compartment change. The test group $(n=5)$ was pretrained in the first session with a behavioral paradigm that varied from the following avoidance training. The 6-sec ongoing tone and the 7-sec ongoing footshock were separated by an interstimulus interval of $8 \mathrm{sec}$ and the option to interrupt the footshock stimulus by change of shuttlebox compartment (Bischof, Stark, Wagner, \& Scheich, 2000). The pretraining session was followed by the two above-mentioned avoidance training sessions.

Although there was no difference in their avoidance performances and reaction times (Figure 6), the distinction in the AR frequency between the groups (Figure 7) demon- strates the utility of a behavioral fine analysis (Stark, Bischof, Wagner, \& Scheich, 2000).

\section{DISCUSSION}

The introduction of the described tone-coding system of PC status signals in combination with The Observer 3.0 (Noldus, 1991; Noldus et al., 2000) generated an ODF with event markers. These events were used to structure a training session into numbered trials, which were further analyzed by the custom-made Excel macros. This method allowed a powerful and time-saving semiautomatic collection and analysis of behavioral data. This useful completion for The Observer system can be adapted without considerable efforts. The behavioral fine analysis using the AR data revealed differences in the behavioral strategies between the groups that were not found by exclusively analyzing the standard parameters, such as conditioned responses or reaction time.

For more detailed information and technical requests concerning the hardware or for further information on the behavioral research, readers should contact the authors. 


\section{REFERENCES}

Bischof, A., Stark, H., Wagner, T., \& Scheich, H. (2000). The inhibitory influence of an acquired escape strategy on subsequent avoidance learning of gerbils. Neuroscience Letters, 281, 175-178. Noldus, L. P. J. J. (1991). The Observer: A software system for collection and analysis of observational data. Behavior Research Methods, Instruments, \& Computers, 23, 414-429.

Noldus, L. P. J. J., Trienes, R. J. H., Hendriksen, A. H. M., Jansen, H., \& JANSEN, R. G. (2000). The Observer Video-Pro: New software for collection, management, and presentation of time-structured data from videotapes and digital media files. Behavior Research Methods, Instruments, \& Computers, 32, 197-206.

Stark, H., Bischof, A., Wagner, T., \& Scheich, H. (2000). Stages of avoidance strategy formation in gerbils are correlated with dopaminergic transmission activity. European Journal of Pharmacology, 405, 263-275.

(Manuscript received April 21, 2000; revision accepted for publication August 5, 2001.) 